\title{
A novel low-voltage and low-power bandgap voltage reference
}

\author{
XING Xiao Ming ${ }^{1, a}$, LI Jian Cheng ${ }^{1, b}$ and Yang $\mathrm{Li}^{1, \mathrm{c}}$ \\ ${ }^{1}$ School of Electronic Science and Engineering, National University of Defense Technology, \\ Changsha, China \\ a13298657014@163.com, ${ }^{b}$ lijc_hh@126.com, ${ }^{c}$ yangli713@126.com
}

Keywords: bandgap voltage reference; low-power; low-temperature coefficient; subthreshold region

\begin{abstract}
The paper proposes a novel low-voltage and low-power bandgap voltage reference, which has superior performance such as low-voltage, low-power and low-temperature coefficient. The simulation based on 0.18-um CMOS process of TSMC. The proposed circuit consists of current source subcircuit, a bipolar transistor and a proportional-to-absolute-Temperature (PTAT) voltage generators, the PTAT voltage generator compensates for negative temperature dependence of a base-emitter voltage in a PNP bipolar transistor, and the voltage with zero temperature coefficient can be generated. Moreover, all of the MOSFETs work in subthreshold region, as a result, the low-voltage and low-power is achieved. The supply voltage is $1 \mathrm{~V}$,and it can also work normally from $0.8 \mathrm{~V}$ to $4 \mathrm{~V}$, while the output voltage of the circuit is $0.58 \mathrm{~V}$,with maximal power dissipation less than $38 \mathrm{nA}$. The temperature coefficient is about $14.5 \mathrm{ppm} /{ }^{\circ} \mathrm{C}$ under temperature from $-40^{\circ} \mathrm{C}$ to $80^{\circ} \mathrm{C}$, that means a better temperature stability. So the performance of the proposed circuit is excellent.
\end{abstract}

\section{Introduction}

A bandgap voltage reference (BGR)is an important block of power management chip, widely used in analog integrated circuits, digital integrated circuits and mixed-signal integrated circuits[1]. The purpose of the voltage reference is to establish a dc voltage that will not be affected by process and voltage ,so that to provide a stable reference voltage for the circuit [2]. It can improve the stability of the circuit system. With the rapid development of wireless communications, the wide application of portable electronic products(e.g cellphone, the ipad),the technical index of the low voltage and low power are getting more and more attention of IC designer and manufacturer. As the power and stability of BGR plays a decisive role for the whole circuit system's performance, the low-voltage and low-power BGR is required. However, For the limitation of the process, the operational amplifier's output voltage in traditional BGR is about $1.2 \mathrm{~V}$,which means the input voltage is higher so that it will waste the power greatly. That, coupled with the complicated circuit structure, make it very difficult to design a low power and low disorder operational amplifier. For above reasons, the low-voltage and low-power bandgap voltage reference is a trend in the future[3].

To solve these problems, this paper proposes a novel bandgap voltage reference circuit that has low voltage and low power. Since the difference of the gate-source voltage of two subthreshold MOSFETs has a positive temperature coefficient, we can design a proportional- to- absolute-

Temperature (PTAT) voltage generators. On the other hand, the base-emitter voltage $V_{B E}$ of the single bipolar has a negative temperature coefficient, which can counteract with the PTAT voltage generators, then the voltage with zero temperature coefficient can be generated by regulating PTAT voltage generators and the aspect ratio(=W/L)of the MOS transistor, Specific implement method will be presented in the following sections. To keep the power dissipation to nanowatts, all of the MOSFETs work in subthreshold region.

\section{Proposed bandgap reference structure}

Fig. 1 shows the complete schematic diagram of the proposed bandgap reference circuit. The circuit consists of four blocks, i.e. start_up circuit,current source subcircuit, voltage divider and 
reference voltage generator.

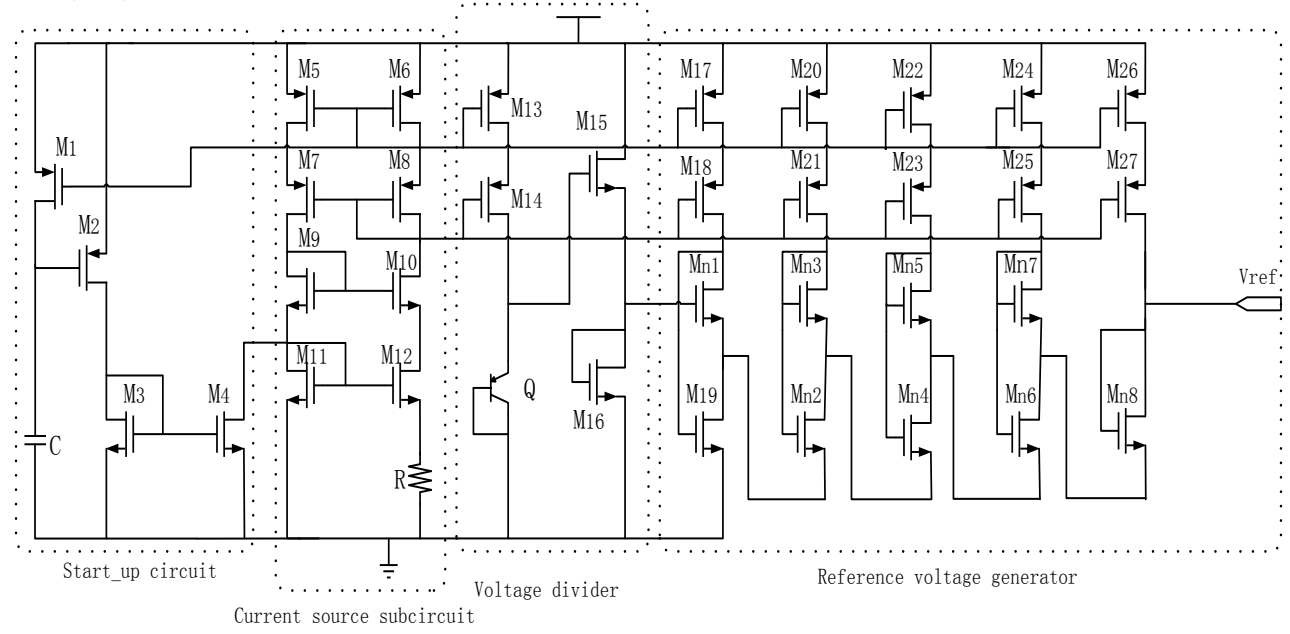

Fig.1 Schematic of proposed bandgap reference circuit

Start_up circuit consisting of transistors $\mathrm{M}_{1} \sim \mathrm{M}_{4}$ and capatior $\mathrm{C}$,is used to avoid the stable state in the zero bias condition. Current source subcircuit containing transistors $M_{5} \sim M_{12}$ and resistor $R$ are used to generates current which can provide bias current for following circuit, the power supply rejection ratio(PSRR) operates well with the cascode structure. The voltage divider circuit divides the base-emitter voltage $V_{B E}$, and makes the output voltage $V_{B E}$ that is a half of $V_{B E}$. The reference voltage generator generates the PTAT voltage. By connecting the output voltage $V_{B E}^{\prime}$, the voltage with zero tempreture coefficient can be generated. The operating principles of the circuits are as follows.

Current source subcircuit. Firstly, considering the subthreshold characteristic of long channel MOS devices, the subthreshold current $I_{D}$ can be expressed as

$$
I_{D}=K I_{0}\left(\exp \frac{V_{G S}-V_{T H}}{\eta V_{T}}\right)\left(1-\exp \frac{-V_{D S}}{V_{T}}\right) .
$$

where $\mathrm{K}(=\mathrm{W} / \mathrm{L})$ is the aspect ratio of the transistor; $I_{0}\left(=\mu C_{O X}(\eta-1) V_{T}^{2}\right)$ is the pre-exponetial factor of the subthreshold current; $\mu$ is the carrier mobility; $C_{O X}$ is the gate-oxide capacitance; $\eta$ is the subthreshold slope factor; $V_{T}\left(=k_{B} T / q\right)$ is the thermal voltage; $k_{B}$ is the Boltzmann constant; $\mathrm{T}$ is the absolute temperature, and $\mathrm{q}$ is the elementary charge ; $V_{T H}$ is the threshold voltage of a MOSFET[4]; For $V_{D S}>4 V_{T}$, the current $I_{D}$ is almost independent of $V_{D S}$ and almost equal to

$$
I_{D}=K I_{0}\left(\exp \frac{V_{G S}-V_{T H}}{\eta V_{T}}\right) \text {. }
$$

From (2),we can get the expression

$$
V_{G S}=\eta V_{T} \ln \left(\frac{I_{D}}{K I_{0}}\right)+V_{T H} .
$$

The structure of the current source subcircuit is shown in Fig.2. Both $M_{n 1}$ and $M_{n 2}$ is operating in subthreshold region. 


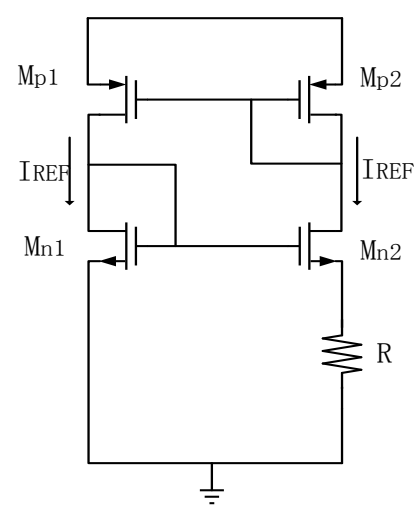

Fig. 2 Schematic of current souce sub-circuit

From Fig.2, gate-source $V_{G S 1}$ in $\mathrm{M}_{\mathrm{n} 1}$ is equal to the sum of gate-source $V_{G S 1}$ in $\mathrm{M}_{\mathrm{n} 2}$ and the voltage of resistor R,i.e.,

$$
\begin{gathered}
V_{G S 1}=V_{G S 2}+V_{R} . \\
V_{R}=I_{R E F} \bullet R .
\end{gathered}
$$

As two branch current of the current mirror consists of $\mathrm{M}_{\mathrm{p} 1}$ and $\mathrm{M}_{\mathrm{p} 2}$ is equal, $I_{R E F}$ can be expressed according to (3) and (4) as

$$
\begin{aligned}
& I_{R E F}=\frac{V_{G S 1}-V_{G S 2}}{R} \\
& =\frac{\eta V_{T} \ln \frac{I_{D}}{K_{1} I_{0}}+V_{T H}-\eta V_{T} \ln \frac{I_{D}}{K_{2} I_{0}}-V_{T H}}{R} \\
& =\frac{\eta V_{T}}{R} \ln \frac{K_{2}}{K_{1}} .
\end{aligned}
$$

where $\mathrm{K}_{1}\left(=\mathrm{W}_{1} / \mathrm{L}_{1}\right)$ is the aspect ratio of the transistor $\mathrm{M}_{\mathrm{n} 1}, \mathrm{~K}_{2}\left(=\mathrm{W}_{2} / \mathrm{L}_{2}\right)$ is the aspect ratio of the transistor $\mathrm{M}_{\mathrm{n} 2}$. By regulating the vaule of $\mathrm{K}_{1}, \mathrm{~K}_{2}$ and $\mathrm{R}$, we can obtain $10 \mathrm{nA}$ current.

voltage divider. The bipolar accepts the current through a current mirror and generates a base-emitter voltage $V_{B E}$, which is expressed as

$$
V_{B E}=V_{T} \ln \left(\frac{I}{I_{s}}\right) \text {. }
$$

where $I_{s}$ is the saturation current of the bipolar. Because $V_{B E}$ decreases linearly with temperature,(6) can be simplified as

$$
V_{B E}=V_{B G R}-\gamma T \text {. }
$$

where $V_{B G R}$ is the bandgap voltage of the silicon (i.e.,typ.1.1-1.2)[5]; $\gamma$ is the temperature coefficient of the $V_{B E}$. In order to obtain low output voltage $V_{B E}$, the circuit uses voltage divider circuit.Fig.1 shows the proposed sub-BGR circuit, that is based on the source-follower circuit. By regulating the aspect ratio of the transistor $\mathrm{M}_{16}$, that $V_{B E}$ is made equal to the half of the $V_{B E}$,i.e.

$$
V_{B E}^{\prime}=\frac{1}{2} V_{B E}=\frac{1}{2} V_{B G R}-\frac{1}{2} \gamma T \text {. }
$$

Reference voltage generator. As the current of the current source subcircuit is at the level of nanoampere, due to the effect of current mirror, the whole MOSFETs of the reference voltage generator operates in subthreshold region. From (5), the difference of the gate-source voltage $\Delta V_{G S}$ of two subthreshold MOSFETs can be expressed as

$$
\Delta V_{G S}=V_{G S 1}-V_{G S 2}=\eta V_{T} \ln \frac{K_{2}}{K_{1}} .
$$

Therefore, by making the ratio of $\mathrm{K}_{2} / \mathrm{K}_{1}$ larger than 1 ,PTAT voltage can be obtained . However, 
because the ratio of $\mathrm{K}_{2} / \mathrm{K}_{1}$ is included in a logarithmic function as shown in Eq.(9),temperature coefficient of the voltage is too small to cancel out the negative coefficient dependence of $V_{B E}$. According to the simulation analysis, it can be achieved by connecting four pairs in cascade. From Fig.1, the reference voltage $V_{\text {ref }}$ can be obtained.

$$
V_{\text {ref }}=V_{B E}^{,}+V_{G S 2}-V_{G S 1}+V_{G S 4}-V_{G S 3}+V_{G S 6}-V_{G S 5}+V_{G S 8}-V_{G S 7}
$$

From Eqs.(8) and (9) , this also can be expressed as

$$
V_{\text {ref }}=\frac{1}{2} V_{B G R}+\left(-\frac{1}{2} \gamma+\sum_{i}^{4} \eta \frac{k}{q} \ln \left(\frac{K_{2 i-1}}{K_{2 i}}\right)\right)
$$

From Eqs.(11), by regulating the ratio of $\mathrm{K}_{2 \mathrm{i}-1} / \mathrm{K}_{2 \mathrm{i}}$, the voltage with zero temperature coefficient can be obtained and expressed as

$$
V_{\text {ref }} \approx \frac{1}{2} V_{B G R}
$$

Due to the bandgap voltage of silicon is about $1.1 \sim 1.2 \mathrm{~V}$, the output voltage $V_{\text {ref }}$ is about $0.55 \sim 0.6 \mathrm{~V}$.

\section{Simulation result}

The proposed reference circuit shown in Fig. 1 has been designed using 0.18-um CMOS process of TSMC. It is tested and verified by using the platform of the Cadence Spectre[6]. Fig.3 shows the measured output voltage $V_{\text {ref }}$ as a function of VDD, with supply voltage VDD as a parameter. The average output voltage $V_{\text {ref }}$ is $0.58 \mathrm{~V}$ in the power range of 0 to $4 \mathrm{~V}$, as the output voltage $V_{\text {ref }}$ keeps stable in the power range of 0.8 to $4 \mathrm{~V}$.

Fig.4 shows the corresponding power dissipations of bandgap reference, which is about $37 \mathrm{nW}$ when the supply voltage VDD was $1 \mathrm{~V}$.

Fig.5 shows measured output voltage $V_{\text {ref }}$ as a function of temperature, with supply voltage VDD as a constant. The voltage variation is within $1 \mathrm{mV}$ in the temperature range from -40 to $80^{\circ} \mathrm{C}$,so the temperature coefficient is $14.5 \mathrm{ppm} /{ }^{\circ} \mathrm{C}$.

Fig. 6 shows the power supply rejection ratio(PSRR) at room temperature with a $1 \mathrm{~V}$ power supply in a Frequency range from $0.01 \sim 1 \mathrm{GHz}$. From Fig.6, it revealed the PSRR was $-42 \mathrm{~dB}$ at $100 \mathrm{~Hz}$.

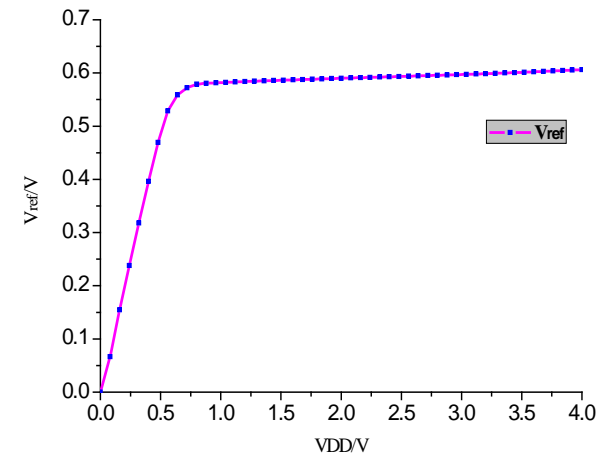

Fig. 3 Simulated output voltage Vref as a function of VDD

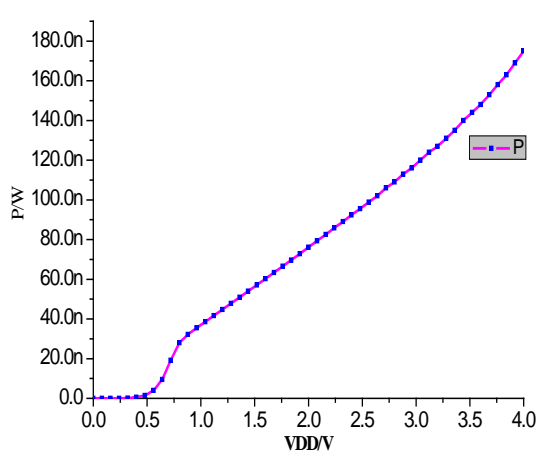

Fig.4 Simulated power dissipations of bandgap reference as a function of VDD 


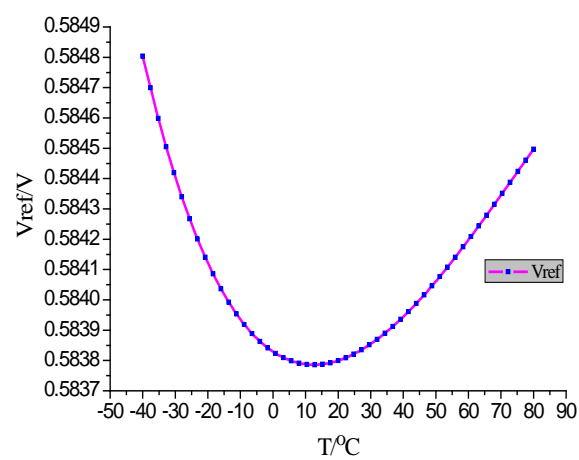

Fig.5 Simulated voltage reference with temperature

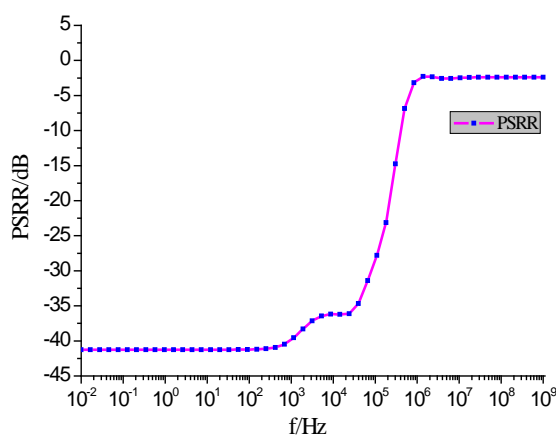

Fig.6 Simulated PSRR as a function of Frequency

Table1summaries circuit performances in comparison with other reported bandgap voltage references[8]-[11] in recent years. From Table 1, the proposed bandgap voltage reference operates with ultralow power dissipation and low temperature coefficient in comparison with other bandgap voltage references with the same process. Moreover, the proposed circuit shows very good performance in terms of process variations in sensitivity and the more wide voltage range. The proposed circuit is therefore useful as a voltage reference for power-aware LSIs[7].

Table 1 comparsion of other lower-power CMOS voltage references

\begin{tabular}{cccccc}
\hline & This work & {$[8]$} & {$[9]$} & {$[10]$} & {$[11]$} \\
\hline CMOS Technology & $0.18-u m$ & $0.18-u m$ & $0.18 u m$ & $0.18-u m$ & $0.18-u m$ \\
VDD $(\mathrm{V})$ & $0.8-4.0$ & $>0.9$ & $0.85-2.5$ & $0.7-1.8$ & $1-5$ \\
Vref (V) & 0.58 & 0.66 & 0.58 & 0.55 & 0.3 \\
Temp. range $\left({ }^{\circ} \mathrm{C}\right)$ & $-40-80$ & $0-150$ & $40-85$ & $-40-120$ & $-23-77$ \\
TC $\left(\mathrm{ppm} /{ }^{\circ} \mathrm{C}\right)$ & 14.5 & 40 & 50 & 114 & 72.6 \\
Power dissipation $(\mathrm{uW})$ & 0.037 & 473 & 0.06 & 0.06 & 1.48 \\
PSRR(dB) & $-41 @ 1 \mathrm{KHz}$ & - & - & - & - \\
\hline
\end{tabular}

\section{Conclusion}

This paper proposes a novel bandgap voltage reference circuit, which has many advantages ,e.g. low power dissipation, low supply voltage and low temperature coefficient. The appropriate use of connecting negative temperature coefficient circuit and positive temperature coefficient circuit in cascade, generated the voltage with zero temperature coefficient. The output voltage of the proposed circuit is $0.58 \mathrm{~V}$, with only $8 \mathrm{mV}$ deviation under the working power from $0.8 \mathrm{~V}$ to $4 \mathrm{~V}$, and the temperature coefficient is $14.5 \mathrm{ppm} /{ }^{\circ} \mathrm{C}$, with only $1 \mathrm{mV}$ deviation under the temperature from -40 to $80^{\circ} \mathrm{C}$. Because of the characteristics of the low-voltage and low-power, the proposed circuit is corresponding to the development trend of modern integrated circuits.

\section{References}

[1] Qiang Zhao,Mng Lu,Jian Zhang.Design and analysis of the bandgap voltage reference source[J] Electronic Sci.\&Tech.2012:1-3.

[2] BEHZAD R. Design of Analog CMOS Integrated Circuit [M]. Xian: Xian Jiaotong University Press,2003.

[3] Paulr.Gray,Paul.Hurst. Analysis and design of analog integrated circuits[M].Beijing:Higher education press,2011.

[4] Sergio Franco.Design with Operational Amplifiers and Analog Integrated Circuits[M].Xian: Xian Jiaotong University Press,2010. 
[5] Tingcun Wei,Yingmei Chen, Zhengfei Hu.Analog CMOS Integrated Circuit Design[M].Beijing: Tsinghua University Press,2010.

[6] Lenian He,Yi Wang.Design and simulation of Analog CMOS Integrated Circuit[M].Beijing:the science press,2011.

[7] Ken Ueno,Tetsuya Asai.A 300nW,15ppm/ ${ }^{\circ} \mathrm{C}, 20 \mathrm{ppm} / \mathrm{V}$ CMOS voltage reference circuit consisting of subthreshold MOSFETs[J]. IEEE Solid-State Circuits.2009:2047-2053.

[8] Tetsuya H,Ken U ,Nobutaka K.A COMS bandgap and sub-bandgap voltage reference circuits for nanowatt power LSIs[C].IEEE Asia Sol Sta Circ Conf.Beijing,China.2010:2-6.

[9] Huimin Zhang,Yun Zhou,Kai Yuana,and Yadong Jiang.A voltage reference circuit with ultra-low power using subthreshold and body effect techniques[J]. IEEE Solid-State Circuits.2010:46-49.

[10]Yuji Osaki, Tetsuya Hirose.etc.1.2V supply,100-nW,1.09-V bandgap and 0.7-V supply,52.5-nW,0.55-V subbandgap reference circuit for nanowatt CMOS LSIs[J]. IEEE Solid-State Circuits.2013:1-9.

[11]Babacan S D,Molina R,Katsaggelos A K,Bayesian Compressive Sensing Using Laplace Priors[J]. IEEE Trans on Image Processing.2010:53-63. 